REVISTA CIENTÍFICA RURAL

ISSN: $1413-8263 \quad 2525-6912$
Revista

Técnico-Científica
Q

EDIUACAM

urcomp

\title{
PRODUÇÃO DE FRUTOS PARTENOCÁRPICOS DE ABÓBORA HÍBRIDA “TETSUKABUTO” SOB APLICAÇÃO DE 2,4-D
}

\author{
${ }^{1}$ Mirian Nomura, ${ }^{2}$ Henrique Pereira Franco, ${ }^{3}$ Estevam Matheus Costa, ${ }^{4}$ Bruno de Moraes \\ Nunes, ${ }^{5}$ Eleusa Maria Ferreira Rocha
}

\begin{abstract}
${ }^{1,4}$ Doutorandos em Agronomia Pela Universidade Federal de Uberlândia e professores na Universidade do Estado de Minas Gerais, ${ }^{2}$ Engenheiro Agrônomo pela Universidade do Estado de Minas Gerais, ${ }^{3}$ Mestrando em Ciências Agrárias - Agronomia pelo Instituto Federal Goiano, Campus Rio Verde, ${ }^{5}$ Doutora em Genética pela Universidade de São Paulo e professora na Universidade do Estado de Minas Gerais
\end{abstract}

RESUMO: "Tetsukabuto" é um híbrido interespecífico, resultado do cruzamento entre linhagens de Cucurbita máxima e C. Moschata, e devido à esterilidade da flor masculina, é necessária a utilização de outra espécie para a polinização. Outra forma de se obter a frutificação é através do uso de fitohormônios, produzindo frutos partenocárpicos. O objetivo do trabalho foi avaliar a produção de frutos de "Tetsukabuto" com o uso de diferentes dosagens de 2,4-D aplicadas nas flores femininas. O trabalho foi desenvolvido na Universidade do Estado de Minas Gerais entre março e julho de 2016, no delineamento experimental de blocos casualisados

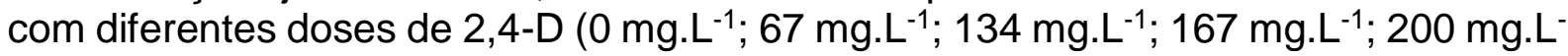
${ }^{1}$ ), quatro repetições e seis plantas por parcelas. Avaliou-se, a quantidade de flores que produziram frutos, o peso dos frutos maduros e seu diâmetro longitudinal, os dados foram submetidos à análise de variância pelo teste $F$ e as médias dos tratamentos foram ajustadas às equações de regressão. A concentração de $140 \mathrm{mg}$. L${ }^{1}$ de 2,4-D foi a mais eficaz na produção de frutos partenocárpicos de Tetsukabuto.

Palavras-chave: Fitohormônio, partenocarpia, moranga japonesa.

\section{PRODUCTION OF PARTENOCARPIC FRUITS OF TETSUKABUTO HYBRID PUMPKIN UNDER 2,4-D APPLICATION}

ABSTRACT: "Tetsukabuto" is an interspecific hybrid, a result of the cross between Cucurbita maximal lineages and C. Moschata, and due to the sterility of the male flower, it is necessary to use another species for pollination. Another way to obtain fruit is through the use of phytohormones, producing parthenocarpic fruits. The objective of this work was to evaluate the production of "Tetsukabuto" fruits with the use of different dosages of 2,4-D applied in the female flowers. The study was carried out at the State University of Minas Gerais between March and July 2016, in the experimental design of randomized blocks with different doses of 2,4-D (0 mg.L -1, $67 \mathrm{mg} . \mathrm{L} \mathrm{-1,} 134 \mathrm{mg} \mathrm{L-}$ 1, $167 \mathrm{mg} . \mathrm{L}-1,200 \mathrm{mg} \cdot \mathrm{L}-1)$, four replicates and six plants per plot. It was evaluated the amount of flowers that produced fruits, the weight of the mature fruits and their longitudinal diameter, the data were submitted to analysis of variance by the $F$ test and the means of the treatments were adjusted to the regression equations. The 
concentration of $140 \mathrm{mg} \cdot \mathrm{L}-1$ of 2,4-D was the most effective in the production of parthenocarpic fruits of Tetsukabuto.

Keywords: Phytohormone, parthenocarpy, Japanese strawberry.

\section{INTRODUÇÃO}

A abóbora híbrida ou "Tetsukabuto", popularmente conhecida como "Cabotiá" ou moranga japonesa, foi produzida originalmente no Japão e devido a boa adaptação às condições locais, esses híbridos têm grande importância econômica no Brasil. É um híbrido interespecífico, resultado do cruzamento entre linhagens selecionadas de moranga (Cucurbita máxima Duch.), utilizadas como genitores femininos, e linhagens de abóbora (C. moschata Duch.), utilizadas como genitores masculinos.

As plantas possuem expressão sexual monóica, ou seja, produzem flores femininas e masculinas na mesma planta, mas por ser um híbrido interespecífico apresentam flores masculinas estéreis, necessitando do plantio de plantas polinizadoras para a frutificação. Assim, para que ocorra a produção de frutos, é imprescindível a disponibilidade de pólen originário de outras espécies de abóbora ou de moranga (FILGUEIRA, 2007). Este fato pode representar um problema, pois parte da área de plantio não poderá ser cultivada com o "Tetsukabuto", e sim com a espécie polinizadora, e há, também, uma dependência de insetos para realizar a polinização das flores.

Outra forma de se obter a frutificação é através do uso de hormônio sintético, sendo os mais utilizados a base de 2,4-D (Aminamar, Aminol, Capri, DMA, U-46, 2,4D Amina, dentre outros), produzindo, desta forma, frutos partenocárpicos. Segundo Ashton e Crafts (1973), o herbicida 2,4-D (ácido 2,4- diclorofenilacético) é um regulador de crescimento que possui efeito análogo ao hormônio auxina.

As flores pulverizadas com auxinas (AIA) podem induzir a formação do fruto a partir do desenvolvimento da parede do ovário, sem que ocorra a fecundação (MIRANDA, 2012). Segundo Pereira (2016), quando a produção de frutos de "Tetsukabuto" ocorre por meio da reprodução sexuada há uma taxa de "pegamento" dos frutos de 25 a $50 \%$ e com a reprodução assexuada, utilizando-se a auxina sintética, como o 2,4-D, há um acréscimo na produção de frutos de 60 a $80 \%$. 
Pesquisas desenvolvidas pela Embrapa Hortaliças mostram que as dosagens de 200 a 250 ppm de 2,4-D promoveram maiores produtividades em abóboras híbridas do tipo "Tetsukabuto". Nesse trabalho foi observado que valores acima do recomendado atuam de maneira inversa à auxina natural (AIA) existente no interior das plantas, proporcionando uma completa desregulação nos principais processos metabólicos (PEREIRA; MENEZES, 1999).

Estudos realizados por Amarante; Macedo (2000) e Pasqualetto et al. (2001) com abóbora e Godoy e Cardoso (2004) com pepino, sobre a produção de frutos por partenocarpia com uso de 2,4-D, demonstram ser possível a obtenção de frutos com o uso deste fitoregulador, porém a dose utilizada pode variar com as condições ambientais. Os produtos comerciais possuem diferentes concentrações de 2,4-D e, desta forma, torna-se importante ajustes na concentração da solução dos fitoreguladores ou calibragem da dose de aplicação por flor (AMARO et al., 2014). Estudos sobre a produção de híbridos do fruto tipo "Tetsukabuto", via assexuada com uso de reguladores vegetais, indicando qual dose mais apropriada a essa cultura, pode ser uma alternativa de melhores rendimentos aos produtores (FERREIRA et al., 2017).

Assim, objetivou-se neste trabalho quantificar as características correlacionadas a produtividade de abobora híbrida "Tetsukabuto" por meio de partenocarpia induzida por fitoregulador 2,4-D do grupo auxina.

\section{MATERIAL E MÉTODOS}

O experimento foi desenvolvido na área experimental do campus UEMG, unidade Ituiutaba, com uma altitude média de $604 \mathrm{~m}$ localizada no município de Ituiutaba - MG, cujas coordenadas geográficas são 18 58' 16.48" Latitude S e 49²6' 52.07" Longitude W. O clima em Ituiutaba, de acordo com classificação de Koppen é do tipo Aw, tropical chuvoso, com temperatura e precipitação média anual de $24{ }^{\circ} \mathrm{C}$ e $1.470 \mathrm{~mm}$, respectivamente (ABRÃO, 2007).

$O$ delineamento experimental foi em blocos casualizados (DBC), com cinco tratamentos (T1 - utilizou-se apenas água destilada; T2 - concentração de $67 \mathrm{mg} . \mathrm{L}^{-1}$ de ácido 2,4-dicloro fenoxiacético; T3 - $134 \mathrm{mg} \cdot \mathrm{L}^{-1} ; \mathrm{T} 4$ - $167 \mathrm{mg} \cdot \mathrm{L}^{-1} ; \mathrm{T} 5$ - $200 \mathrm{mg.L^{-1 }}$ ) e quatro repetições, contendo seis plantas por parcela experimental. Para aplicação do 2,4-D usou-se o produto 2,4-D Nortox, produzido pela empresa Nortox S/A, herbicida seletivo sistêmico do grupo químico ácido ariloxialcanóico, o qual foi utilizado 
na preparação da solução de fitorregulador sendo cada concentração diluida. O produto comercial 2,4-D Nortox, que contem $670 \mathrm{~g}$ de ingrediente ativo (ácido 2,4dicloro fenoxiacético) por litro foi diluído de modo a obter as concentrações estabelecidas para o experimento, para T1 utilizou-se apenas água destilada, para T2 utilizou-se $0,10 \mathrm{ml}$ do produto, em T3 foram $0,2 \mathrm{ml}$, para T4 $0,25 \mathrm{ml}$ e T5 foram 0,30 $\mathrm{ml}$ do produto diluídos em um litro de água.

Incialmente foi coletada amostra de solo e enviada ao laboratório de solos da UEMG, unidade Ituiutaba, para a realização de análise de solo. Posteriormente, foram produzidas mudas da moranga híbrida em bandejas de poliestireno expandido de 128 células preenchidas com substrato da marca Bioplant ${ }^{\circledR}$, foi realizado a semeadura no dia 15/04/2016. Foram plantadas seis plantas por parcela experimental, com o espaçamento de 2 metros $(\mathrm{m})$ entre linhas e 1,5 metros entre plantas totalizando 120 plantas. Foi realizado o preparo do solo e foram feitas as covas de $20 \times 20 \times 20$ centímetros $(\mathrm{cm})$ onde foram aplicados os fertilizantes minerais: $20 \mathrm{~g}$ de ureia, $88 \mathrm{~g}$ de superfosfato simples, $20 \mathrm{~g}$ de cloreto de potássio por cova e, também, incorporado a terra o fertilizante orgânico (esterco bovino) na dosagem de 2 litros por cova, e após, realizou-se o transplantio das mudas 17 dias após a emergência das plântulas. A adubação de cobertura foi dividida em duas aplicações, sendo uma após 15 dias (20 g/cova de ureia) e a segunda após 30 dias do plantio ( $20 \mathrm{~g} /$ cova de Ureia).

Foram realizadas duas aplicações do inseticida Evidence ${ }^{\circledR}$ na concentração de 1g.L-1 para o controle de pulgão aos 21 e 41 dias após o transplantio das mudas (DAT) além de uma aplicação do fungicida Amstar Top ${ }^{\circledR}$ aos 35 e 65 DAT. O controle de plantas infestantes foi realizado por meio de capinas manuais, deixando a cultura livre de competição.

Após a abertura das primeiras flores femininas, que se deram aos 29 DAT, foram realizadas as pulverizações das soluções de 2,4-D dos tratamentos. Foram aplicados $2 \mathrm{~mL}$ da solução de cada tratamento no interior de cada flor feminina. A aplicação foi realizada com pulverizador manual sempre na parte da manhã entre às 6 horas e às 10 horas por ser a hora que as flores estão mais receptivas, onde eram anotadas todas as flores que foi realizado a pulverização da solução em cada parcela durante 22 dias seguidos.

Devido à diferença de maturação dos frutos, foram realizadas duas colheitas para avaliação, a primeira foi realizada 90 dias após a abertura das primeiras flores, a segunda foi realizada 10 dias após a primeira. Foram avaliadas as seis plantas de 
cada parcela, sendo que todos os frutos foram colhidos. Os parâmetros analisados foram os seguintes: porcentagem frutificação, peso médio dos frutos, e diâmetro longitudinal dos frutos.

A porcentagem de frutificação, ou seja, flores que produziram frutos comercializáveis foi realizada contando-se diariamente as flores pulverizadas com os tratamentos, e após dez dias da aplicação foi verificado se houve a produção de frutos partenocárpicos. O peso dos frutos foi verificado por meio da pesagem logo após a colheita com utilização de balança digital. Logo em seguida, foi medido o diâmetro longitudinal dos frutos com o auxílio de fita métrica.

Os dados foram submetidos a análise de variância ao nível de $5 \%$ de probabilidade (Teste F), para todas as características avaliadas, ajustaram-se equações de regressão, havendo, para todos os parâmetros avaliados, ajuste ao modelo polinomial quadrático. As análises foram feitas com auxílio do software SISVAR e SPSS.

\section{RESULTADOS}

Verificou-se que, com utilização da dosagem de 2,4-D a $139 \mathrm{mg} \cdot \mathrm{L}^{-1}$, alcançouse a maior indução de frutificação, ou seja, com esta dosagem, 69,48\% das flores pulverizadas produziram frutos (Figura 1). 


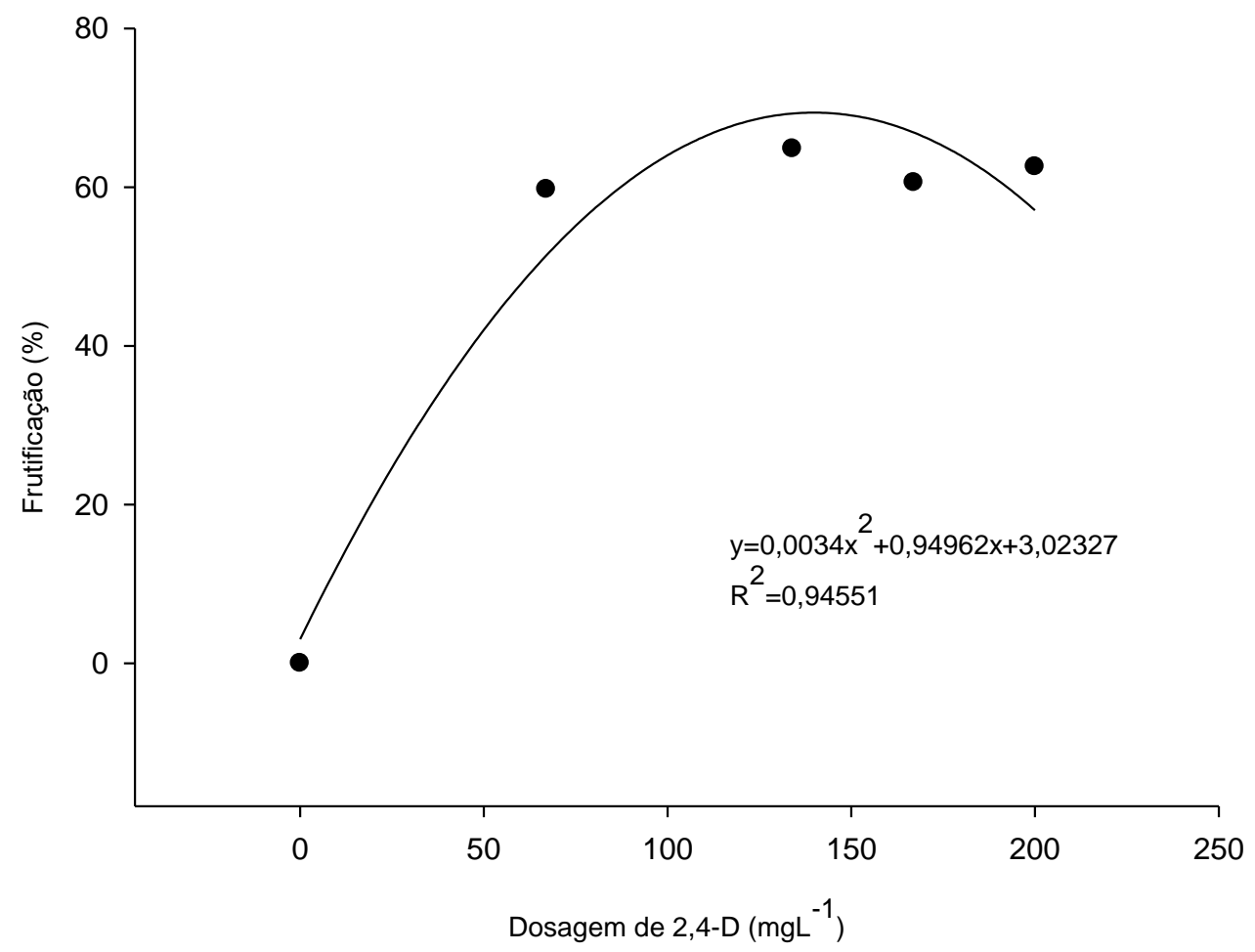

Figura 1 - Estimativa da porcentagem de frutificação com a aplicação de diferentes dosagens de 2,4-D. Figure 1 - Estimation of the percentage of fruiting with the application of different dosages of 2,4-D.

Quanto ao peso dos frutos, houve aumento de valores à medida que a dose de 2,4-D foi sendo elevada, até $141,5 \mathrm{mgL}^{-1}$. Esse resultado demonstrou que esta dose estimulou a produção de frutos mais pesados, com mais de 1,08 $\mathrm{kg}$ de peso (Figura 2). A partir desta dosagem, há uma diminuição progressiva do peso dos frutos. 


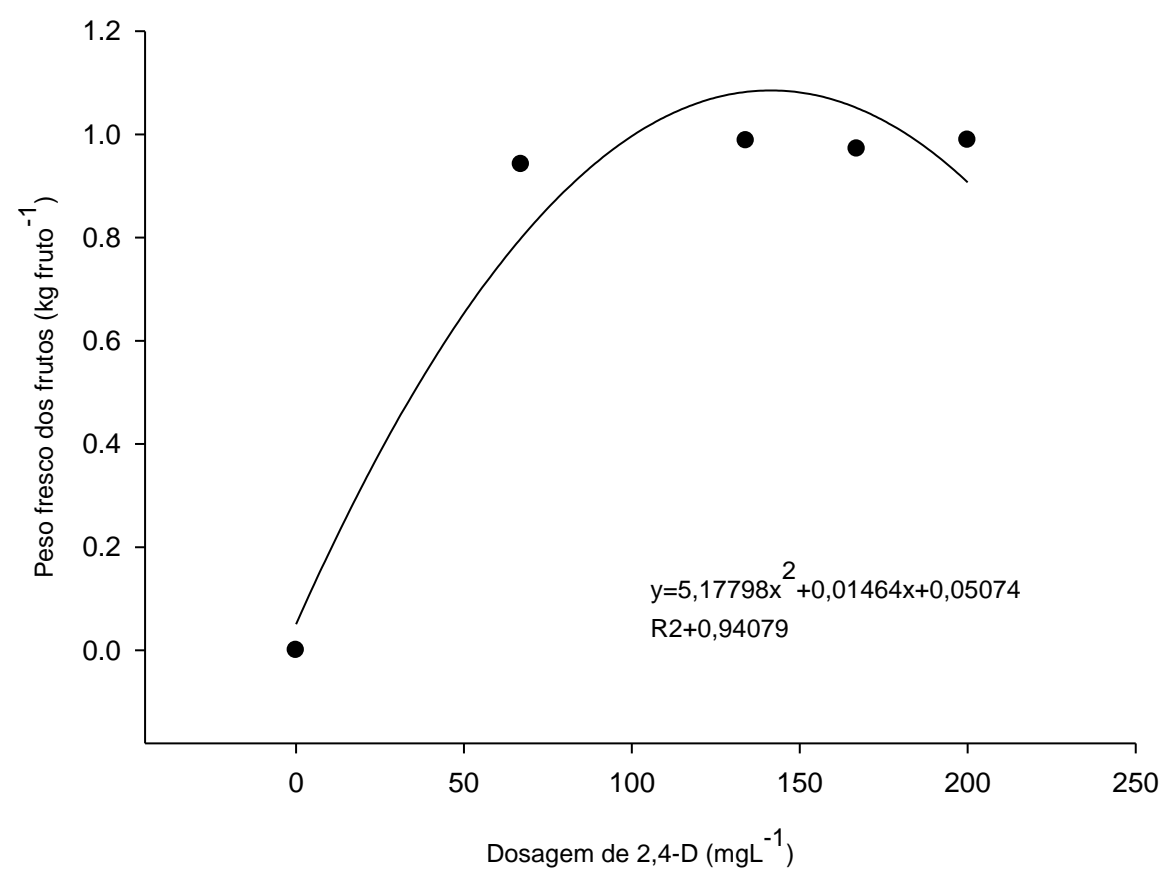

Figura 2 - Estimativa peso dos frutos com a aplicação de diferentes dosagens de 2,4-D. Figure 2 - Estimation of fruit weight with the application of different dosages of 2,4-D.

Em relação ao diâmetro dos frutos de "Tetsukabuto", verificou-se que com o aumento da dosagem até $140 \mathrm{mg} \cdot \mathrm{L}^{-1}$ do produto testado, ocorreu um aumento gradual do diâmetro dos frutos, nessa dosagem, foi estimada a produção de frutos de $25,5 \mathrm{~cm}$ de diâmetro (Figura 3).

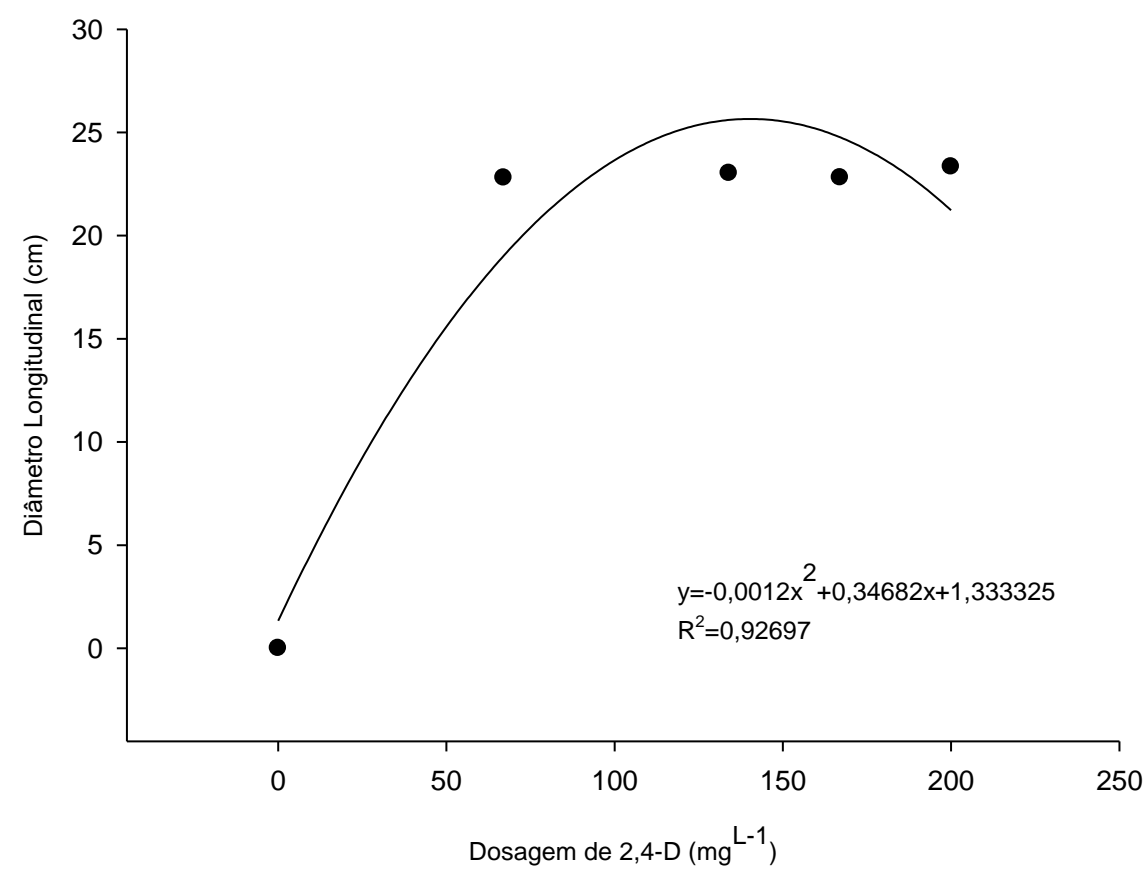

Figura 3 - Estimativa diâmetro longitudinal dos frutos com a aplicação de diferentes dosagens de 2,4-D. Figure 3 - Estimated longitudinal diameter of fruits with the application of different dosages of 2,4-D.

Revista Científica Rural, Bagé-RS, volume21, №2, ano 2019 


\section{DISCUSSÃO}

O resultado do aumento da indução de frutificação foi similar ao de Oliveira et al. (2002), ao avaliarem a indução da frutificação em moranga híbrida com 2,4-D, testando as dosagens $50,100,200$, e $400 \mathrm{mg} \cdot \mathrm{L}^{-1}$, aplicadas no interior e na parte externa da flor feminina, concluíram que melhores respostas na indução da frutificação foram alcançadas com a dosagem de 100 a $200 \mathrm{mg}^{-\mathrm{L}^{-1}}$ de 2,4-D no interior da flor

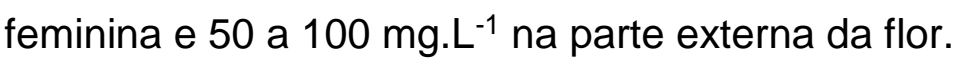

Em relação ao peso do fruto fresco se assimila ao de Pasqualetto et al. (2001), avaliando dosagens variando de 0 a $200 \mathrm{mg}^{-L^{-1}}$ de 2,4-D aplicados na flor feminina de "Tetsukabuto" e mostrando que as diferentes dosagens não influenciaram no número de frutos por planta e no peso médio de frutos por planta, porém a utilização de 150 mg.L-1 de 2,4-D contribuiu significativamente no aumento do peso total de frutos por planta, em 68,10 \% em relação à testemunha. Pereira et al. (2012), ao avaliarem dosagens de 2,4-D de 0,60, 120, 180 e $240 \mathrm{mg}^{-\mathrm{L}^{-1}}$, verificaram que a dose de 195, 8 $\mathrm{mg.L-1}$, proporcionou frutos de maior tamanho, com um valor estimado de $17,7 \mathrm{~cm}$. Portanto, a variação no peso está relacionada ao tamanho dos frutos, conforme a circunferência aumentou (Figura 3 ) houve aumento no peso, o que demonstra que, as dosagens de 2,4-D, provavelmente, não interferiram na espessura da polpa.

O diâmetro dos frutos, de $25,5 \mathrm{~cm}$, produzidos com a dosagem de $140 \mathrm{mg} . \mathrm{L}^{-1}$ apresentam padrão semelhante aso frutos produzidos por via sexuada (NASCIMENTO et al., 2008), porém, vale ressaltar que quando há produção de frutos por partenocarpia não há necessidade de plantio de uma espécie polinizadora, assim a área cultivada com abóboras híbridas é maior.

Desta forma, a dosagens próximas a $140 \mathrm{mg} . \mathrm{L}^{-1}$ proporcionaram as melhores estimativas em todos os parâmetros analisados, a partir deste valor há um decréscimo devido ao fato do 2,4-D em concentrações maiores do que a auxina natural AIA (ácido indo acético), induz a mudanças metabólicas e bioquímicas, resultando em redução no processo de crescimento. Segundo Miranda (2012), o resultado deste efeito ocorre por que o produto afeta a divisão celular, levando a um crescimento desordenado, o que leva a destruição dos tecidos, podendo afetar ainda a ação da enzima RNApolimerase, o que afetará a síntese de ácidos nucléicos e proteínas, resultando numa alta taxa de divisão celular nos tecidos, podendo levar à morte da planta devido a 
epinastia de folhas e caule, interrupção do floema, o que impede o movimento dos fotoassimilados das folhas para o sistema radicular.

Deve-se ressaltar que a dosagem de $140 \mathrm{mg} \cdot \mathrm{L}^{-1}$ pode ser considerada baixa quando comparada com os resultados de outros trabalhos, Pereira et al. (2012), por exemplo, ao avaliarem dosagens de 2,4-D, variando de 60 a $240 \mathrm{mg} \mathrm{L}^{-1}$, na frutificação de Testsukabuto, concluíram que a dosagem de 212,8 $\mathrm{mg} \mathrm{L}^{-1}$, quando comparada à produção sexuada, aumentou o número de frutos produzidos por planta e, também, aumentou a massa dos frutos, refletindo em acréscimo na produtividade da cultura de $123,8 \%$. Oliveira et al. (2015) e Gonçalves et al. (2014), verificaram que a produção de frutos com maior diâmetro foi alcançada com a dosagem de $700 \mathrm{mg} \cdot \mathrm{L}^{-1}$. Miranda (2017), ao avaliar o aumento da indução de frutificação testando doses crescentes do ácido 2,4 Diclorofenoxacético (187,5; 200; 212,5; 225; 237,5; 250 e 262,5 mg.L -1 $^{-1}$ de 2,4-D), concluiu que com o aumento das doses de 2,4-D até $250 \mathrm{mg} \cdot \mathrm{L}^{-1}$ houve aumento da produtividade e peso médio dos frutos.

Os diferentes resultados podem refletir as variadas condições edafoclimáticas a que foram submetidos os experimentos, como também, a diferença de comportamento da planta, no que diz repeito ao material genético, frente ao uso do 2,4-D. O uso de dosagens mais baixas, que consigam dar uma resposta produtiva significativa ao produtor rural, torna-se mais interessante tendo em vista que dosagens muito próximas ao máximo, também estão mais próximas de níveis fitotóxicos.

\section{CONCLUSÕES}

A dosagem de $140 \mathrm{mg} \cdot \mathrm{L}^{-1}$ proporcionou a melhor estimativa de resposta no quesito porcentagem de frutificação, peso médio e diâmetro longitudinal dos frutos.

\section{AGRADECIMENTOS}

À Fundação de Amparo à Pesquisa do Estado de Minas Gerais - FAPEMIG pela concessão de bolsa de Iniciação Científica.

\section{REFERÊNCIAS}


ABRÃO, M. E. G. Determinação dos parâmetros físio-metereológico para estimativa da evapotranspiração potencial,caracterização e classificação do clima em ituiutaba. Dissertação de Mestrado. Belo Horizonte:UEMG. 2007.

AMARANTE, CVT; MACEDO, AF. Frutificação e crescimento de frutos em abóbora híbrida 'Tetsukabuto' tratada com alfanaftalenoacetato de sódio. Horticultura Brasileira, n18, 212-214. 2000.

AMARO, G. B.; PINHEIRO, J. B.; LOPES, J. F.; CARVALHO, A. D. F de C.; MICHEREFF FILHO, M.; VILELA, N. J. Recomendações técnicas para o cultivo de abóbora híbrida do tipo japonesa. Circular técnica, EMBRAPA. Brasília, DF. 2014.

ASHTON, F.M.; CRAFTS, A.S. Mode of action of herbicides. New York: John Wiley, 1973. 504p.

FERREIRA, T.A.; OLIVEIRA, C.R.; CHAVES, P.P.N.; MILHOMENS, K.K.B.; BARROS, H.B.; NASCIMENTO, I.R. Indução da frutificação paternocárpica de frutos em híbrido de abóbora japonesa com 2,4-d sob condições de temperatura elevada. Nucleus, v.14, n.1, p.145-152, 2017.

FILGUEIRA, F. A. R. Novo manual de olericultura: agrotecnologia moderna na produção e comercialização de hortaliças. Viçosa: UFV, 2007.

GODOY, A.R.; CARDOSO, A.I.I. Pegamento de frutos em pepino caipira não partenocárpio sob cultivo protegido com aplicação de ácido naftaleno acético. Bragantia, v.63, n.1, p. 25-29, 2004.

GONÇALVES, J. F. R. F.; MAGALHÃES, C. G.; GONÇALVES, V. D.; VIANA, F. J.; OLIVEIRA, A. F.; DUARTE, A. R.; PORTO, D. M. V. Qualidade dos frutos de abóbora híbrida "Tetsukabuto" submetida a diferentes aplicações de auxina sintética e adubação nitrogenada. Anais... . $\%$ FEPE, 2014.

MIRANDA, F. F. R. NASCIMENTO, I. R. CHAVES, P. P. N. MURAISHI, C. T. DOURADO, D. P. Agronomic characteristics of punpkin fruits of "Tetsukabuto" hybrid as a function of 2,4-D 
doses. Com. Sci., Bom Jesus, v.8, n.4, p.521-525, Oct./Dec. 2017. DOI:

10.14295/CS.v8i4.456.

NASCIMENTO, W.M.; COIMBRA, K.G.; FREITAS, R.A.; BOITEUX, L.S. 2008.

Eficiência de acessos de Cucurbita máxima como polinizadores de abóbora híbrida do tipo "Tetsukabuto". Horticultura Brasileira 26: 540-542.

OLIVEIRA, A. F.; MAGALHÃES, C. G.; GONÇALVES, V. D.; OLIVEIRA, H. R.; VIANA, F. J.; OLIVEIRA, N. B.; MORAIS, M. S. Crescimento dos frutos de abóbora híbrida "Tetsukabuto" em diferentes aplicações de 2,4-D e nitrogênio. Anais... $8^{\circ}$ FEPE, 2015.

OLIVEIRA, V. R.; MASCARENHAS, M. H. T.; PIRES, N. M. Indução da frutificação em moranga-híbrida com ácido 2,4-D. Horticultura brasileira, v.20, n.2, julho, 2002.

PASQUALETTO, A.; SILVA, N. F.; ORDONEZ, G. P.; BARCELOS, R. W. Produção de frutos de abóbora híbrida pela aplicação de 2,4-D nas flores. Pesquisa Agropecuária Tropical, pg 23-27, 2001.

PEREIRA, A. M.; SILVA, G. D.; ALMEIDA, R. R. P.; SILVA, A. B.; QUEIROGA, R. C. F. Frutificação de abóbora "Tetsukabuto" sob aplicação de doses de 2,4-D na época seca em Pombal - PB. Revista Verde de Agroecologia e desenvolvimento sustentável, v.7, n.4, p. 38-43, 2012.

PEREIRA, W. MENEZES, J. E. Avaliação do uso de 2,4-D como fitohormônio na frutificação de moranga híbrida, sob condições de telado. Horticultura Brasileira, v.13, n.1, p. 104. 1999.

PEREIRA, W. Recomendações básicas para a frutificação da abóbora híbrida tipo Tetsukabuto ou Kabutiá. Uso de polinizadores e produtos hormonais. 2001.Comunicado técnico. Disponível em:<https://isla.com.br/cgibin/artigo.cgi?id_artigo=111>. Acesso em: nov., 2016. 